\title{
Amputation an den unteren Extremitäten - Unterschenkel: Indikation, Technik
}

\author{
Lutz Brückner
}

\section{Zusammenfassung}

Die Erhaltung des Kniegelenkes im Rahmen von Amputationen an den unteren Extremitäten ist eine der wichtigsten Voraussetzungen zur Erhaltung guter Rehabilitationsfähigkeit. Die Unterschenkelamputation bietet dafür beste Möglichkeiten, aber nicht jede Methode ist für alle Indikationen, besonders für die am häufigsten vorkommende chronische arterielle Verschlusskrankheit (90\%), geeignet. Es werden die Amputationstechniken, entsprechend ihrer Indikation, von der distalen Syme-Amputation bis zur ultrakurzen Stumpfbildung dargestellt. Wir empfehlen für alle Indikationen, vorausgesetzt die Bildung eines langen Unterschenkelstumpfes ist nicht möglich, die anhand objektiver Parameter entwickelte „Standardisierte Unterschenkelamputation nach Brückner“.

\section{Lower Limb Amputations: Lower Leg: Indications and Technique}

The preservation of the knee joint in the concept of lower extremity amputations is one of the most important prerequisites for obtaining a successful rehabilitation outcome. A transtibial amputation level offers the best possibilities; However, not every surgical procedure is suitable, particularly not for the most common aetiology of chronic arterial occlusive disease (90\%). Surgical techniques are presented according to indications, starting distally with the Syme's amputation through to the structuring of ultrashort transtibial amputation stump levels. In cases where the formation of a long transtibial stump is not possible we recommend for all indications the "standardized transtibial amputation according to Brückner" that was developed on the basis of objective parameters.

\section{Einleitung}

In etwa $90 \%$ der Fälle muss bei rund 55-60000 Amputationen/Jahr [9] in Deutschland wegen chronisch arterieller Verschlusskrankheit mit oder ohne Diabetes mellitus bei oft hoher Multimorbidität der Patienten amputiert werden. In etwa $10 \%$ der Fälle verteilen sich die Amputationsursachen auf traumatisch bedingte bzw. Amputationen wegen Tumor oder in seltenen Fällen wegen Fehlbildungen.

Ein großer Teil der Amputationen betrifft dabei die Unterschenkelamputation, die man immer, sofern eine weiter periphere Amputation nicht möglich ist,

OP-JOURNAL 2007; 23: 200-209

(c) Georg Thieme Verlag KG Stuttgart • New York DOI 10.1055/s-2007-989305 zur Erhaltung des für die Rehabilitation so wichtigen Kniegelenkes anstreben sollte. Auch besonders unter dem Aspekt, dass der durchblutungsgestörte Patient der Gefahr unterliegt, doppelseitig amputiert werden zu müssen. Gerade unter diesem Gesichtspunkt ist es nicht vertretbar, wenn aus Gründen einer evtl. schnelleren Wundheilung womöglich vorzeitig zur Oberschenkelamputation gegriffen wird.

Wir können aufzeigen, dass selbst bei schwersten arteriellen Durchblutungsstörungen die Erhaltung des Kniegelenkes in rd. 93\% der Fälle mit unserer „Standardisierten Amputationstechnik nach Brückner" möglich ist.

In den Fällen, wo wir im Oberschenkel nachamputieren mussten, handelte es sich um so stark multimorbide Patien- ten, dass sie leider in der postoperativen Phase noch im Krankenhaus verstarben.

\section{Equipment}

Wir führen die Unterschenkelamputation mit der Bildung eines hinteren Hautmuskellappens (Burgess, Brückner) im Durchstichverfahren mit dem Amputationsmesser durch. Vorher wurde der kurze vordere Weichteillappen mit dem Skalpell gebildet.

Weitere Instrumente:

- Amputationssäge

- Oszillierende Säge

- Luer

- Feile

- Scheren

- Pinzetten

- Kapselschere aus dem Hüftbesteck

- Knochenfasszange

- Meißel

- Hammer (Technik nach Ertl/Dederich)

- Hohmann-Hebel

- Spülung

- Atraumatisches Nahtmaterial

- Steri strips

\section{Lagerung}

Rückenlage; nach Absetzung des Unterschenkels sollte der Stumpf durch „sterile Rollen" unter dem distalen Oberschenkel zur besseren Übersicht angehoben werden.

Wir benutzen auch gern ein steril eingepacktes Volkmann-Bänkchen.

\section{Blutsperre, Blutleere}

Eine entsprechende Blutsperremanschette haben wir aus Sicherheitsgründen immer an dem Oberschenkel angelegt, aber bei chronisch arterieller Verschlusskrankheit niemals aufgeblasen.

Anders kann es sich bei Amputationen wegen Tumor oder Trauma mit normaler Durchblutung verhalten. Hier ist die 
Blutsperre, so kurz wie möglich, durchaus angezeigt. Eine Blutleere kommt auch hier nicht infrage.

\section{Wo beginnt und wo endet die Unterschenkelamputation?}

Die Frage ist deshalb berechtigt, weil mitunter die Syme-Amputation zu den Rückfußamputationen und nicht zu den Unterschenkelamputationen gerechnet wird.

Da aber die Absetzung im distalen Metaphysenbereich des Unterschenkels erfolgt, zählen wir diese Amputationshöhe zur Unterschenkelamputation.

\section{Gliederung}

I Syme-Stumpf

II Umgekehrter Syme-Stumpf

III Modifizierter Syme-Stumpf

IV Langer Unterschenkelstumpf (distales Drittel)

V Mittellanger Unterschenkelstumpf (mittleres Drittel)

VI Kurzer Unterschenkelstumpf (proximales Drittel)

VII Ultrakurzer Unterschenkelstumpf

\section{Syme-Stumpf (1854)}

\section{Indikation}

- Diabetische, alkoholische Neuropathie

- Osteoarthropathie mit noch ausreichender Durchblutungsstörung, wenn sich mit einer Resektion oder periphereren Amputationen sekundäre Fußdeformitäten bzw. Infekte nicht mehr ausreichend behandeln lassen.

- Bedingt/selten: chronisch arterielle Verschlusskrankheit mit und ohne Diabetes mellitus. Die Durchblutung muss aber das periphere Drittel des Unterschenkels noch erreichen.

- Trauma

- Tumor

- Infektion

\section{Technik}

Die Schnittführung (Abb. 1) erfolgt ventral vom lateralen zum medialen Malleolus bzw. umgekehrt. Dabei werden die Weichteile bis auf den Knochen durchtrennt.

In ähnlicher Weise wird der dorsale (Fersen-)Lappen gebildet. Der Schnitt überquert etwa in Höhe des ChopartGelenkes die Fußsohle.

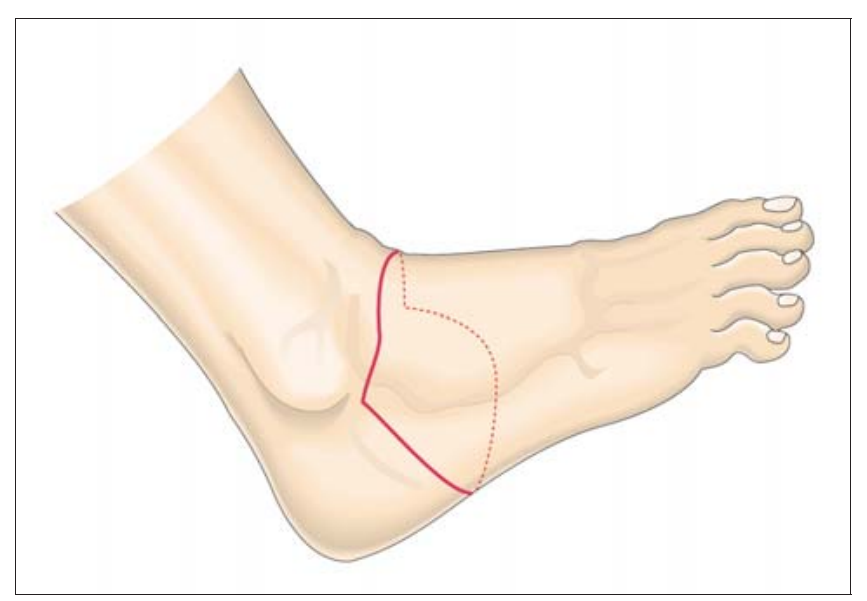

Abb. 1 Schnittführung bei der SymeAmputation. - Aus: Bohne WHO: Atlas of Amputation Surgery, Thieme, 1987 [2].

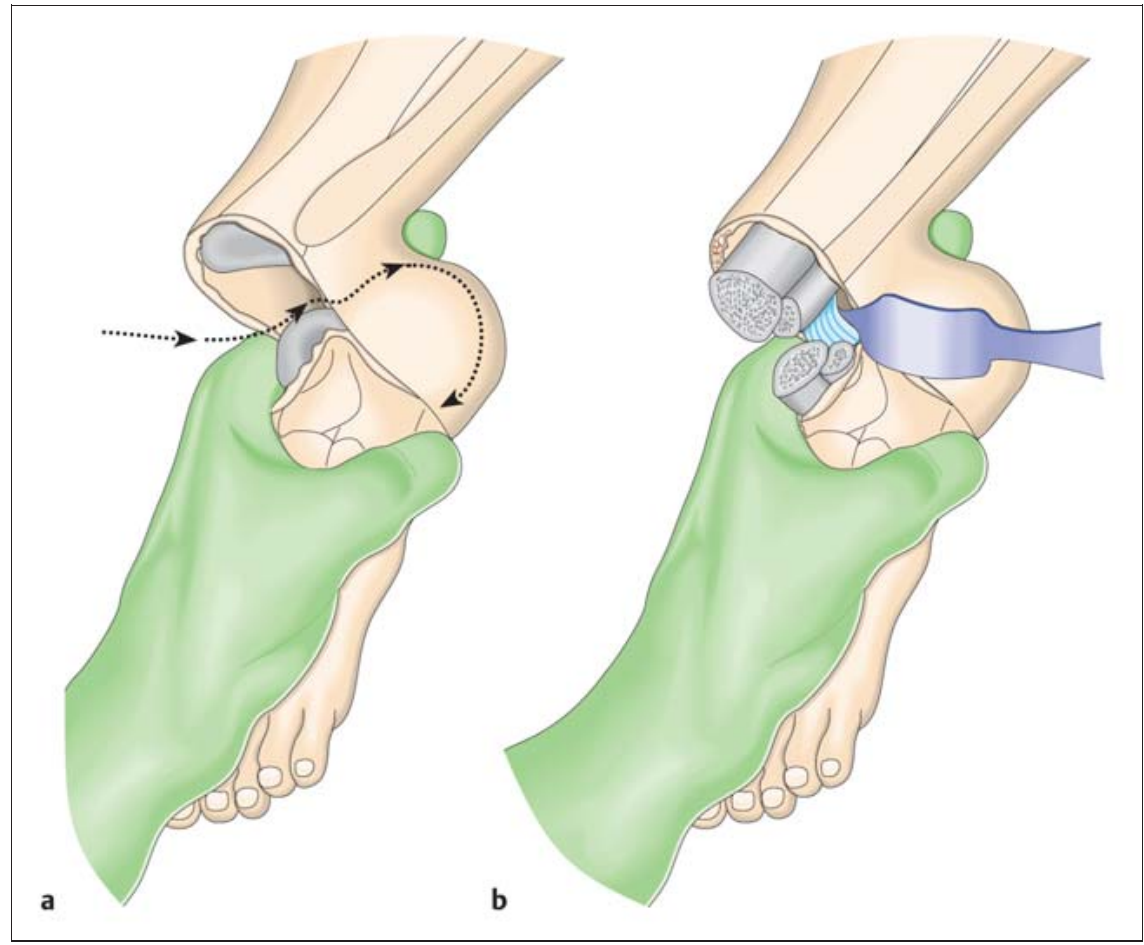

Abb. 2 Unter Plantarflexion und Pro- und Supination wird der Rückfuß herauspräpariert. - Aus: Baumgartner R, Botta P: Amputation und Prothesenversorgung der unteren Extremität, Enke, 1995 [1].

Das Herauslösen des Fußes aus der Knöchelgabel erfolgt durch maximale Plantarflexion und wechselnde Pro- und Supination des Fußes (Abb. 2).

Nur so können die das Fersenbein umgebenden Weichteile angespannt und hart am Knochen abgetrennt werden. Vorsicht ist besonders im Bereich des medialen Malleolus geboten, da gleich hinter ihm die tibialen Gefäße (Arteria und Vena tibiales posterior) verlaufen und bei ihrer Verletzung der für die Deckung und Endbelastbarkeit des Syme-Stumpfes so wichtige Fersenlappen gefährdet ist. Günstig ist deshalb, dass zuerst die Resektionsfläche (Abb. 3) dargestellt und unter Schutz reseziert wird. Die Gefäße sind dann übersichtlicher dargestellt. Wichtig ist, dass die Resektionsfläche im spongiösen Bereich (Endbelastbarkeit) und genau quer zur Belastungslinie liegt (Genu varum, valgum).

Danach kann unter Zug nach unten der Talus und später der Kalkaneus herausgelöst werden. Schwierig wird es noch einmal bei der Durchtrennung der Achillessehne. Hier muss die Perforation der Haut möglichst verhindert werden. Baumgartner und Botta (1995) [1] empfehlen, den Zeigefinger des Operateurs 


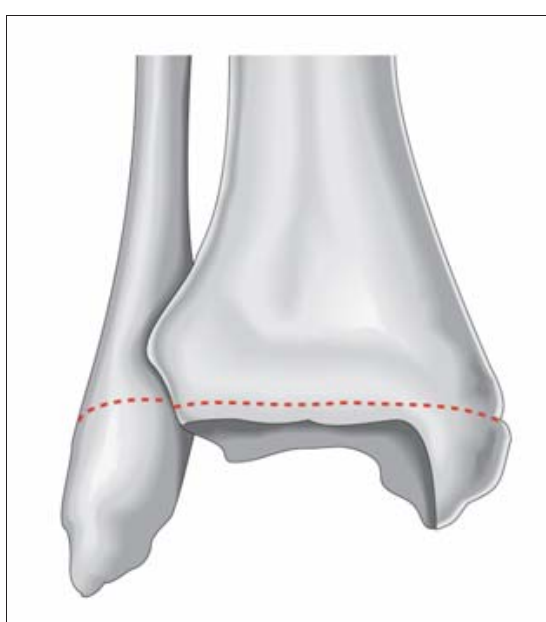

Abb. 3 Knochenresektion. - Aus: Bohne, WHO: Atlas of Amputation Surgery, Thieme, 1987 [2].

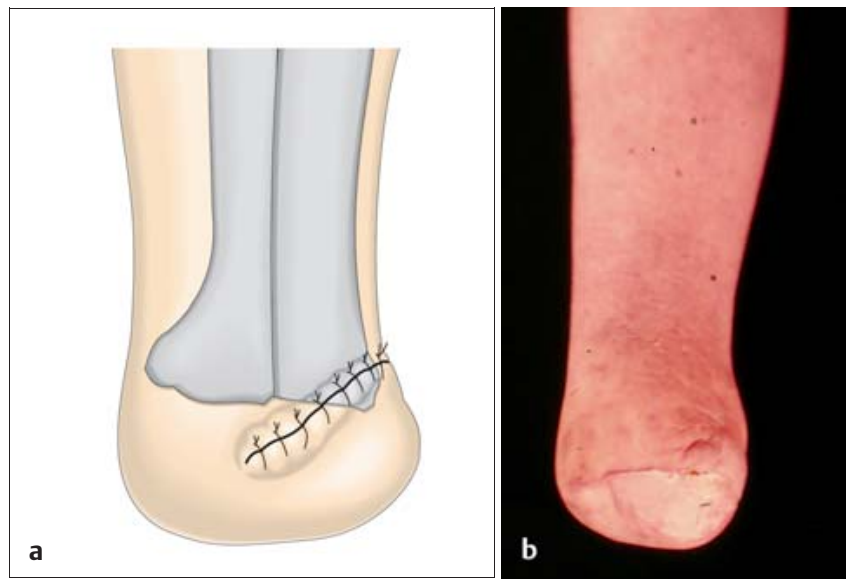

quer über den Ansatz der Achillessehne zu legen und die Achillessehne am besten stumpf mit einer Kocher-Sonde oder einer Cooper-Schere zu umfahren, um dann die Sehne mit dem Skalpell zu durchtrennen.

Nach dem Herauslösen des Fußes wird, sofern erfolgt, die Blutsperre geöffnet, um die Durchblutung des Fersenlappens zu beurteilen.

Der N. tibialis (Cave: Arterie) wird ca. $1 \mathrm{~cm}$ proximal der Resektionsfläche gekürzt.

Tibia und Fibula werden gut abgerundet, um bei Endbelastung von innen keinen Spitzendruck gegen die Weichteile zu erzeugen (Abb.4). Die Sehnen werden gegebenenfalls auf die Höhe der knöchernen Resektionsfläche gekürzt und nicht miteinander vernäht.

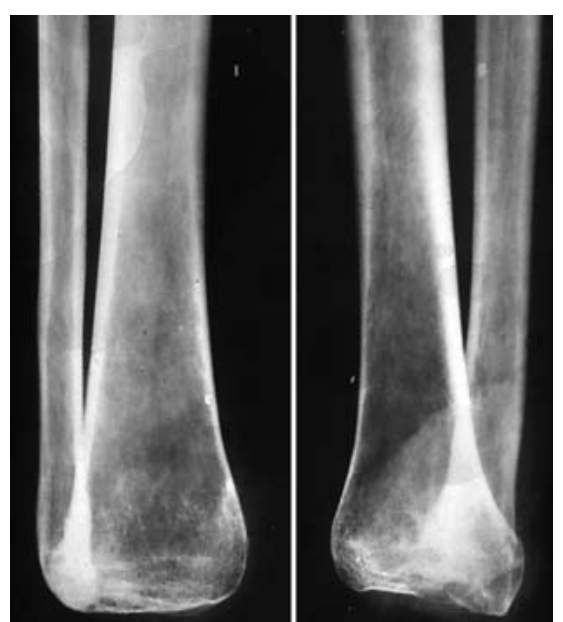

Abb. 4 Gut abgerundete distale Tibia und Fibula. a Hautverschluss ventral. - Aus: Bohne, WHO: Atlas of Amputation Surgery, Thieme, 1987 [2]. b Bildung des Fersenlappens, Narbenverlauf ventral.

Der Fersenlappen wird dann nach Blutstillung nach ventral umgekippt und spannungsfrei mit dem ventralen Lappen vernäht (Abb. 5).

Die unwillkürlich vorhandenen seitlichen „Ohren“ sollen nicht gekürzt werden, um nicht die Basis des Lappens zu verkleinern. Vorher wird ein RedonDrain eingelegt.

\section{Nachbehandlung}

Wichtig ist die postoperative Wickeltechnik, denn sie soll das Abrutschen des relativ lockeren Fersenlappens verhindern. Einige Autoren fixieren deshalb temporär den Fersenlappen mit Kirschner-Drähten, was aber aus unserer Sicht die Infektionsgefahr unnötig erhöht. Bei nicht durchblutungsgestörten Stümpfen erfolgt eine Hochlagerung, ansonsten liegt der Stumpf flach im Bett.

\section{Umgekehrter Syme-Stumpf}

Indikation

Gleiche Indikation wie beim OriginalSyme-Stumpf; aber auch nicht bei geringerer chronisch arterieller Verschlusskrankheit, da der ventrale Lappen schlechter als der dorsale Lappen durchblutet ist.

Im Gegensatz zum Original-SymeStumpf aber bei Verletzung oder Zerstörung des Fersenlappens möglich (Abb. 6).

\section{Unterschied zum Original-Syme-Stumpf}

Langer ventraler und kurzer plantarer/ dorsaler Weichteillappen.

\section{Problem}

Die Endbelastbarkeit wird nicht in jedem Fall erreicht.

Koller et al. (2001) [11] berichten über Endbelastbarkeit bei $45 \%$ ihrer Fälle.

Wir haben in unseren 3 Fällen immer volle Endbelastbarkeit gesehen.

Selbst bei nicht voller Endbelastbarkeit verbleibt aber der lange Hebelarm, was sich biomechanisch günstig auswirkt.

\section{Technik}

Die Schnittführung richtet sich nach dem Fersendefekt, der entfernt werden muss.

Bedingung ist die Erhaltung eines Weichteil-Fußrücken-Lappens (Abb. 7), den man erst kürzen sollte, wenn ein spannungsfreier Verschluss der Wunde überprüft wurde.

Nicht umsonst heißt es „Was weg ist, ist weg“. Ein Verschluss unter Spannung bedeutet aber Misserfolg (Abb. 8).

Die Behandlung von Nerven und Knochen erfolgt analog der Original-SymeAmputation. Die großen Gefäße werden doppelt unterbunden.

\section{Nachbehandlung (Abb.9 und 10)}

Siehe bei Original-Syme-Amputation.

Durch die Amputation kommt es zu einer Verkürzung von $5-7 \mathrm{~cm}$. 

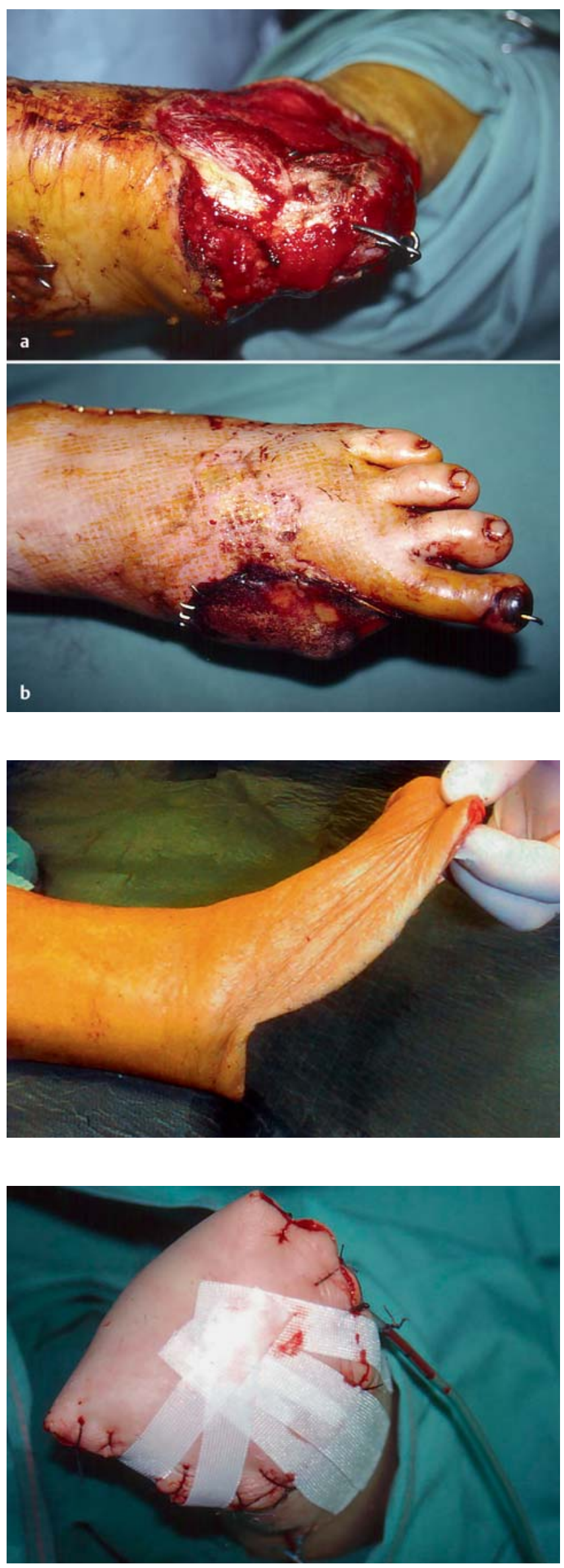
lappens nach dorsal

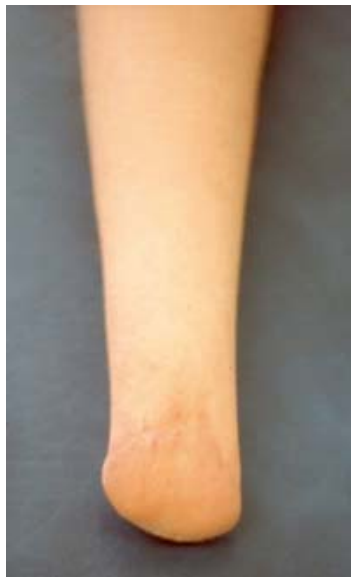

Abb. 9

Syme-Amputation von dorsal.
Abb. 8 Umschlagen des ventralen Hautund Adaptation mit

Naht und Steri strips.

Diese lässt sich aber mit den heute zur Verfügung stehenden prothesentechnischen Mitteln gut ausgleichen. Eine Prothese mit Abstützung oberhalb des Kniegelenkes ist normal nicht notwendig.

\section{Modifizierte Syme-Amputation}

\section{Indikation}

Entspricht der umgekehrten Syme-Indikation.

Hautlappen vor der Kürzung. 


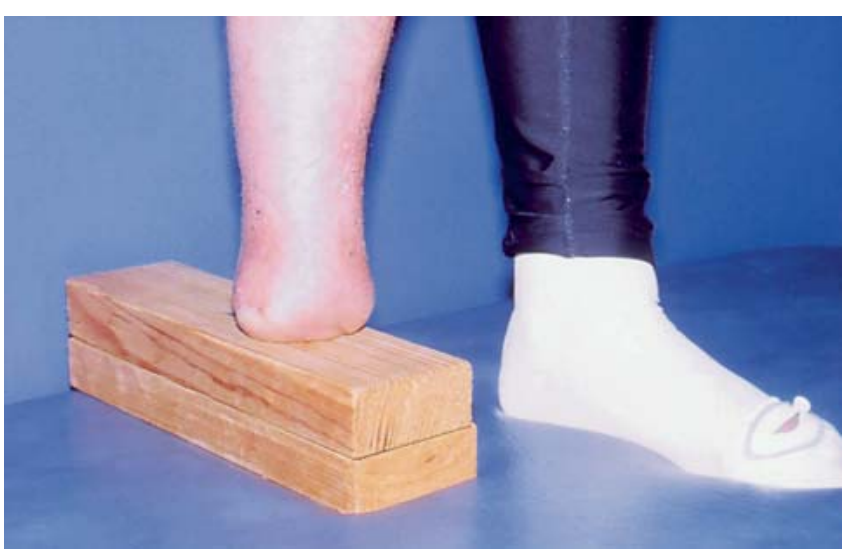

10 Umgekehrte Syme-Amputation, $5 \mathrm{~cm}$ Beinverkürzung, Endbelastung.

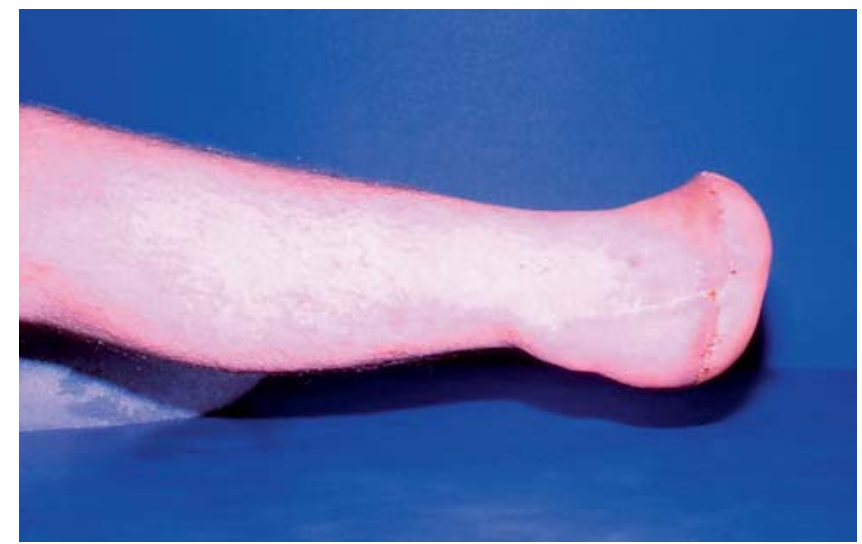

Abb. 11 Modifizierte Syme-Amputation, noch intakte Vorfußsohlenhaut wurde gefäßgestielt unter einen knöchernen Syme-Stumpf gestellt, endbelastbar.

Nachteil

- Die Endbelastbarkeit des Stumpfes ist mit etwa $10 \%$ sehr gering.

- Wegen unzureichender Weichteildeckung (mitunter nur Knochen, Sehnen und Haut) entwickeln diese Stümpfe über die Jahre öfters Durchblutungs- und trophische Störungen (Abb.12), die zur Nachamputation führen. Die Stümpfe sind oft kühl und schmerzhaft. Die Muskelpumpe funktioniert durch die nicht vorhandene Vorspannung [5,8] der Muskulatur schlecht.

\section{Technik}

Bildung eines etwas längeren hinteren und etwas kürzeren vorderen Weichteillappens, oder auch, wenn notwendig, umgekehrt möglich.

Abrundung der ventralen Knochenkante, doppelte Ligatur der großen Gefäße und scharfe Durchtrennung der Nerven bis zu $5 \mathrm{~cm}$ proximal der Knochenresektionsstelle.

\section{Mittellanger Unterschenkel- stumpf (mittleres Drittel)}

\section{Indikation}

- Neuropathie bzw. neuropathische Osteoarthropathie

- Trauma

- Infekt

- Tumor

- Bedingt: chronisch arterielle Verschlusskrankheit mit oder ohne Diabetes mellitus - vorherige genaue Abklärung des Grades der Durchblutungsstörung ist notwendig.

\section{Techniken}

- OP nach Ertl/Dederich (Tumor, Trauma, Infektion)

- OP nach Pinto und Harris (Trauma, Tumor, Infektion, geringere Durchblutungsstörung)

- OP nach Robinson (Tumor, Trauma, Infektion, periphere Gefäßerkrankungen) - bis Kurzstumpf möglich

- OP nach Persson (alle Indikationen angegeben) - bis Kurzstumpf möglich

- OP nach Burgess (alle Indikationen) bis Kurzstumpf

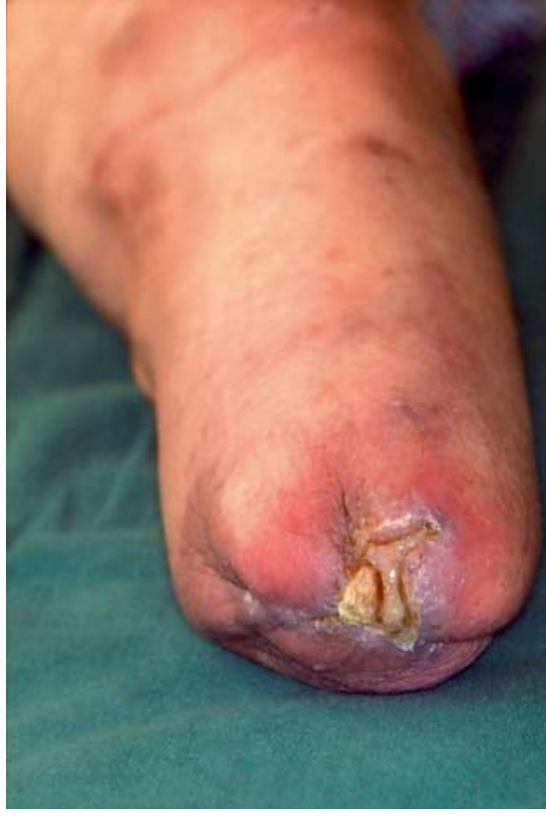

Abb. 12 Langer Unterschenkelstumpf mit trophischen Störungen über die Jahre hinweg, kalt und schmerzhaft.

Operation nach Ertl/Dederich (1949/1987)

Die Operationstechnik ist bei Dederich [7] im Detail nachzulesen.

Das Prinzip ist eine muskel- und knochenplastische Stumpfdeckung.

Dabei wird eine knöcherne Verbindung zwischen Tibia und Fibula über mit Kortikalischips besetztes Periost erreicht. Das Prinzip ist in Abb.13 dargestellt.

Problem

Bei „unsauberem“ Umgang mit dem Periost entstehen knöcherne Ausziehungen am Stumpfende, die in der Prothese dem gewünschten Stumpfendkontakt wegen des Druckes von innen nach außen entgegenwirken.

Operation nach Pinto und Harris (2006)

Technik

Bildung von zwei gleichlangen Hautlappen, die aber auch entsprechend der Situation variiert werden können. Die Lappen werden en bloc mit der Muskulatur gebildet. Wenn möglich, wird von der Tibia ein Periostlappen gebildet, der Stabilität und Vitalität der fibularen Knochenbrücke unterstützt.

Das Fibulasegment, dass zwischen Tibia und Fibula eingeklemmt wird, ist etwa 

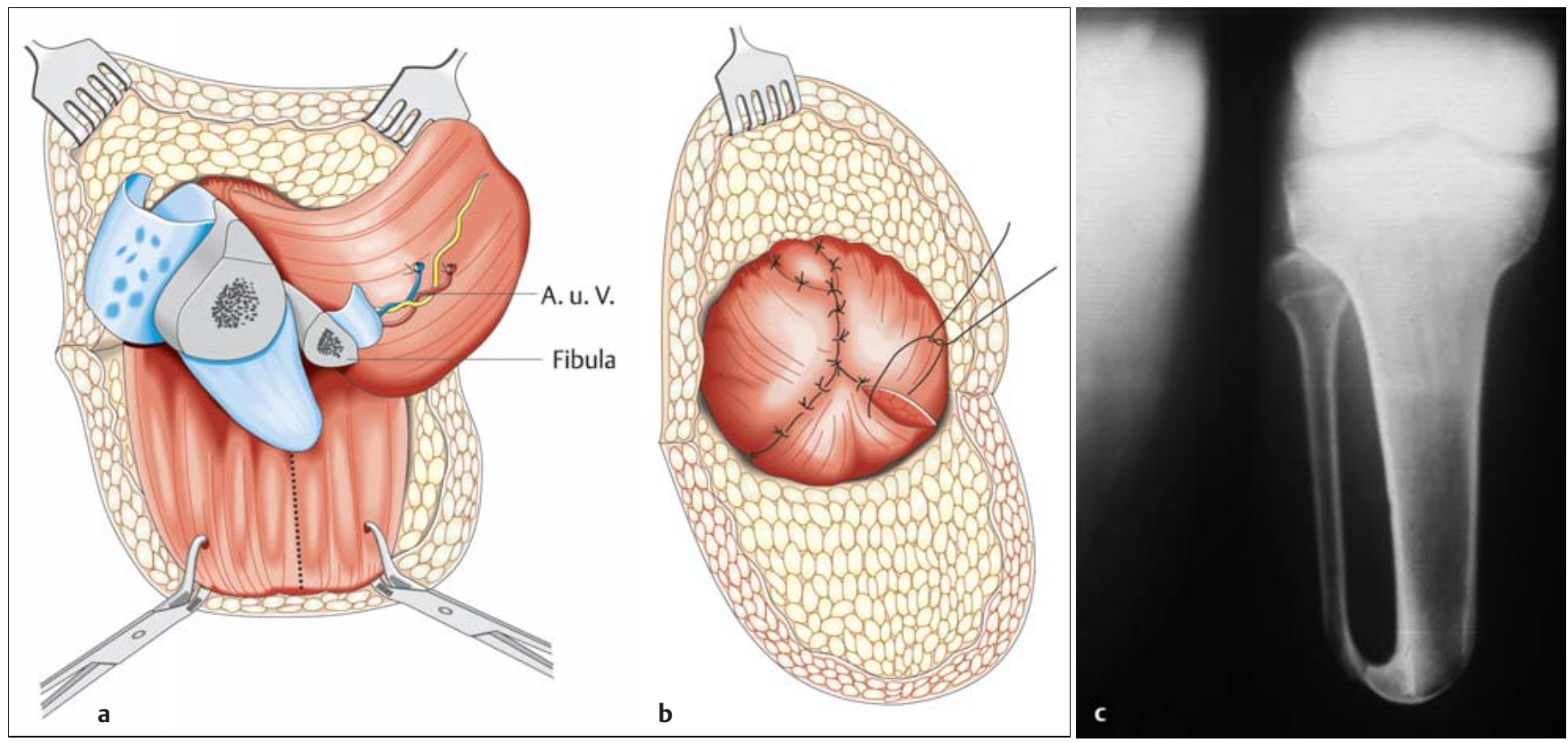

Abb. 13 a bis c Operation nach Dederich mit der Bildung einer Knochenbrücke zwischen Tibia und Fibula. Dazu wird Periost von der Tibia und der Fibula mit anhaftenden Knochenchips so vernäht, dass sich eine Knochenbrücke bilden kann. - Aus: Dederich R: Amputationen der Gliedmaßen, Thieme, 1987 [7].

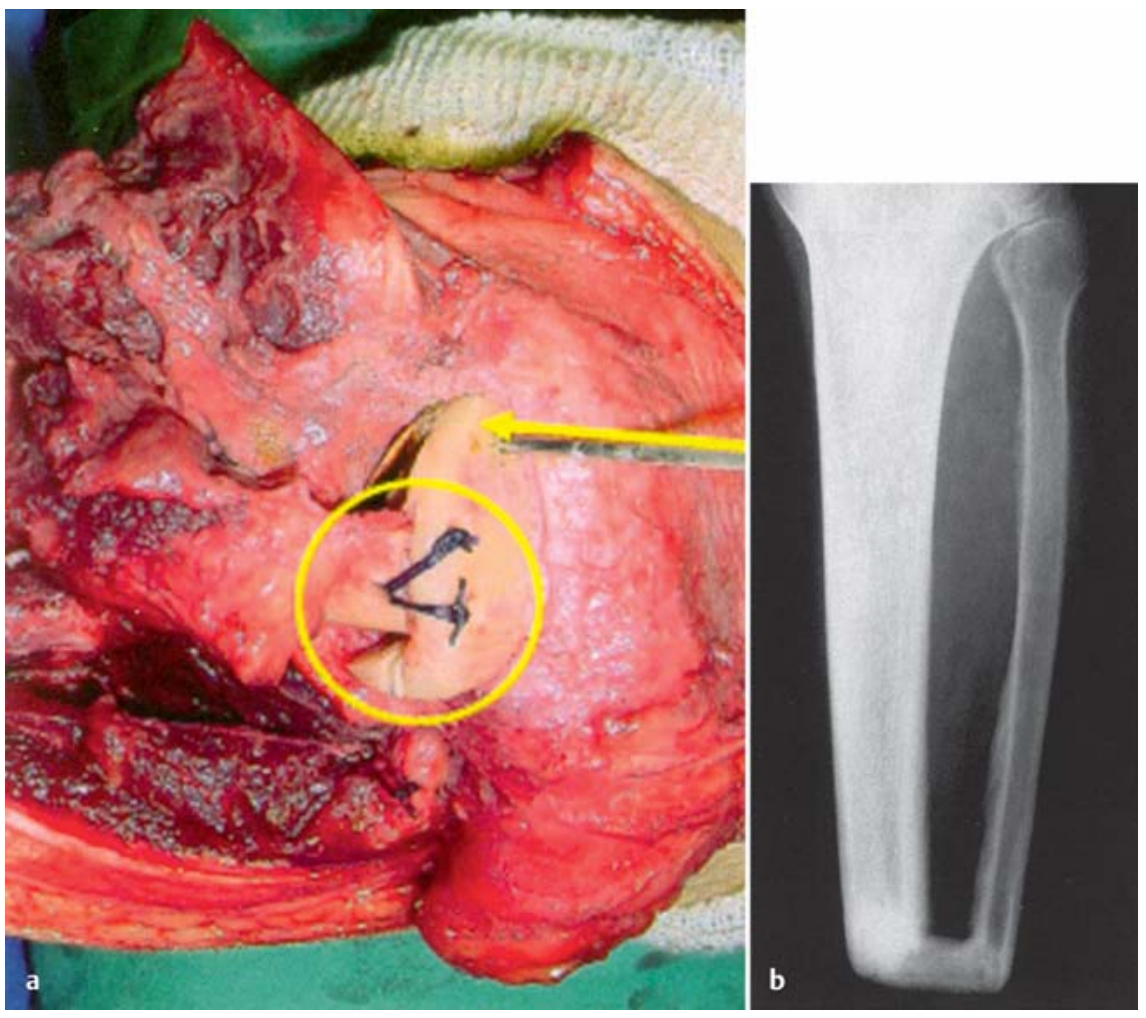

Abb. 14a und b Fibulasegment als Knochenbrücke bei transtibialer Amputation nach Pinto und Harris. a Muskelgestieltes Fibulasegment wird in die Nute der Tibia eingenäht. b Knöchern konsolidiertes Fibulasegment. - Aus: Pinto MAGS et al.: Knochenbrücke mit Fibulasegment bei transtibialen Amputationen, Med Orthop Techn 2006; 126: 45 - 49 [12].

4,5 cm lang. Die dem Fibulasegment angrenzende Muskulatur wird zur besseren Ernährung möglichst belassen. Das zwischen Tibia und Fibula eingesetzte
M. soleus werden auf gleicher Höhe wie die Tibia durchtrennt. Die Gastrocnemii werden vorn an der Tibiakante transossär angeheftet. Die fibulare Muskelgruppe wird dann mit den Gastrocnemii verbunden (Abb.14 b).

Problem

Der Durchbau der Knochenbrücke musste abgewartet und das Prothesentraining konnte im Mittel erst nach 12 Wochen aufgenommen werden.

- Die Zurichtung des Fibulasegmentes, ohne die Verbindung zur Muskulatur zu verlieren, ist aufwendig.

- Für den nicht Erfahrenen besteht die Gefahr der Bildung eines birnenförmigen Stumpfes (überschießende Weichteile). Daraus ergeben sich prothesentechnische Schwierigkeiten.

- Wir gehen davon aus, dass diese Methode, ebenso wie die Methode nach Ertl/Dederich, nicht für die chronisch arterielle Verschlusskrankheit im Stadium IV nach Fontaine geeignet ist.

\section{Vorteil}

- Bei langen Stümpfen wird durch die breitere knöcherne Stumpfauflagefläche die Stumpfendbelastbarkeit erhöht.

- Gegenüber der Operation nach Ertl/ Dederich sehen die Autoren Vorteile, da eine größere Strecke der Tibia (etwa $7 \mathbf{c m}$ ) skarifiziert werden muss, 
was aus ihrer Sicht nicht immer möglich scheint.

Operation nach Robinson (1995)

Prinzip

Schrägsagittale Lappenbildung, die dem arteriellen Hautzufluss im oberen Drittel des Unterschenkels über die Arterien des N. saphenus und des N. surales gerecht wird (Abb.15). Die Narbe liegt zwischen Fibula und Tibia.

Nachteil

Wir sehen bei dieser Operationsmethode das Hauptaugenmerk auf die Hautdurchblutung gelegt. Unsere (siehe unten) Untersuchungen ergaben aber, dass die Durchblutung der Muskulatur bei chronisch arterieller Verschlusskrankheit hauptsächlich für die Entstehung von Wundheilungsstörungen verantwortlich ist. Bei dieser Methode verbleibt aber die Muskulatur im wesentlichen im Stumpf. Wir sehen weiterhin die aus unserer und Gottschalks (2006) Sicht nicht ausreichende physiologische Vorspannung der Muskulatur als Problem an.

\section{Operation nach Persson}

Prinzip

Sagittaltechnik mit zwei seitengleichen Haut-Muskel-Lappen.

Nachteile

Ähnlich der Operation nach Robinson.

Operation nach Burgess (1968)

Prinzip

Es ist das Verdienst von Burgess, der Unterschenkelamputation bei chronisch arterieller Verschlusskrankheit mit der Bildung eines kurzen ventralen Weichteillappens $(1-2 \mathrm{~cm})$ und eines längeren dorsalen Haut-Muskel-Lappens von etwa 13 bis $15 \mathrm{~cm}$ Länge bei einer Tibialänge von etwa $12 \mathrm{~cm}$ zur allgemeinen Verbreitung und damit zum Erhalt des Kniegelenkes verholfen zu haben (Abb.16). Ursprünglich geht diese Technik auf Verduyn (1695) zurück.

Mit dieser guten Methode, auch bei schweren Durchblutungsstörungen, trat aber folgendes Problem auf: Die Muskulatur muss vom Operateur intraoperativ

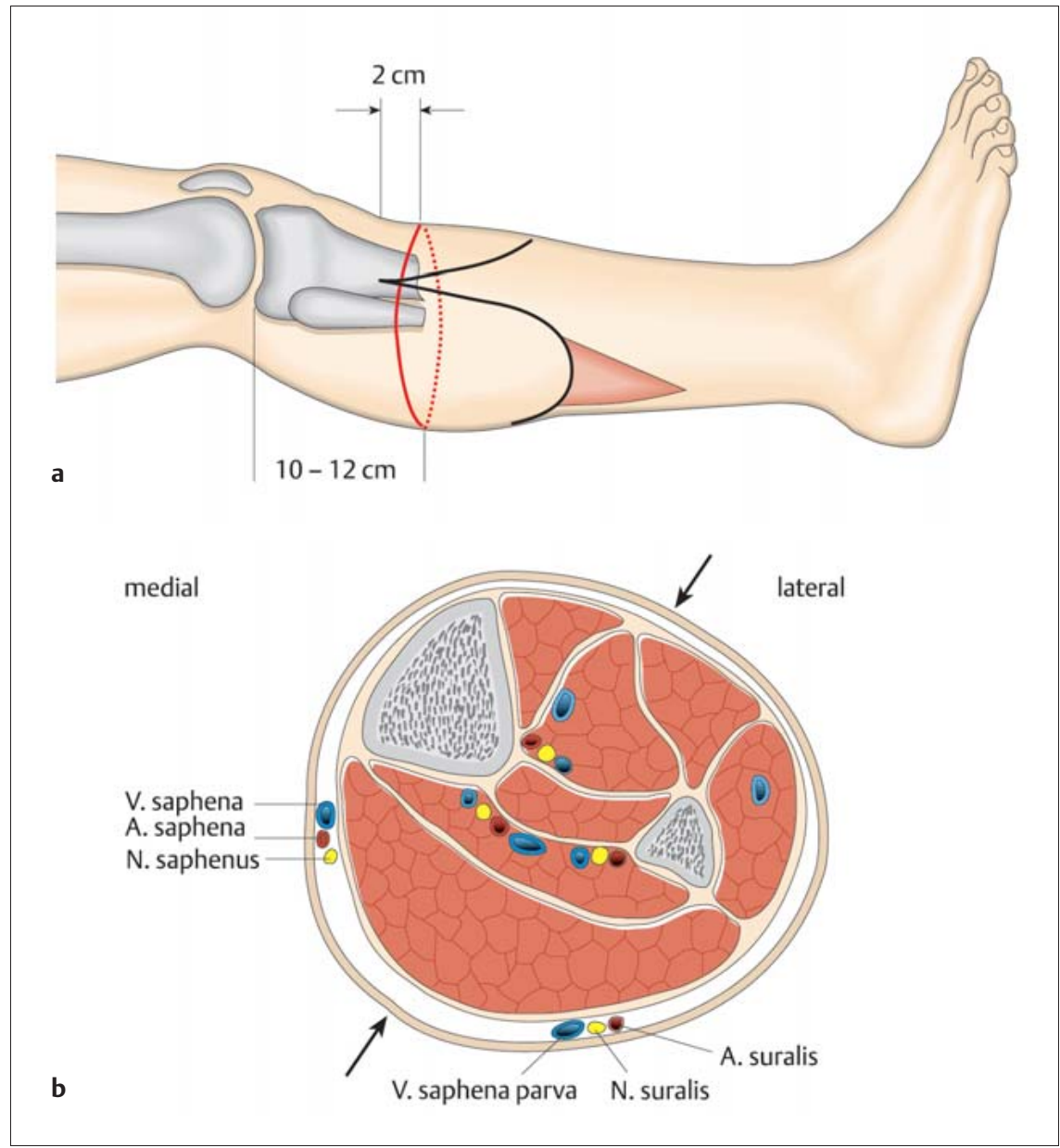

Abb. 15 a und b Schrägsagittale Unterschenkelamputation nach Robinson. a Schnittführung, b Spaltung schrägsagittal zwischen Tibia und Fibula unter dem Belassen von Muskulatur. - Aus: Robinson KP: Schrägsagittale Unterschenkel-Lappen-Amputation, Orthopädie-Technik 1995; 46: $42-49$ [13].

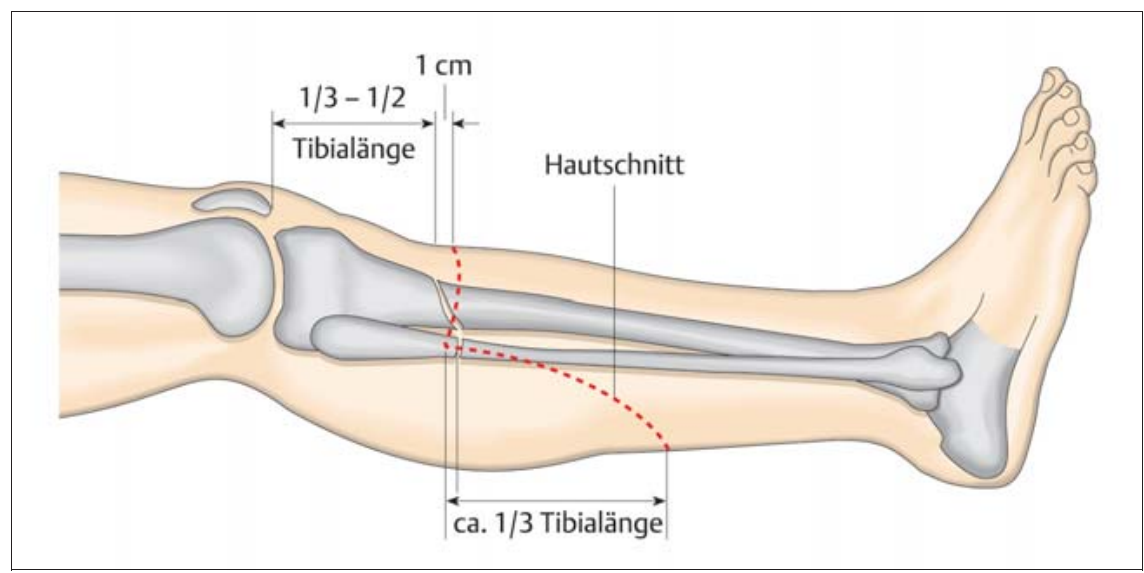

Abb. 16 Operationstechnik nach Burgess, 1968. - Aus: Baumgartner R, Botta P: Amputation und Prothesenversorgung der unteren Extremität, Enke, 1995 [1].

anhand von Farbe, Durchblutung und Kontraktionsverhalten der Muskulatur beurteilt werden. Es hat sich gezeigt, dass das objektiv nicht ausreichend möglich ist. Entsprechend der subjekti- ven Einschätzung der Lebensfähigkeit der Muskulatur müssen aber wichtige Entscheidungen über die Entfernung der einzelnen Muskeln getroffen werden. Dies kann zur Folge haben, dass un- 


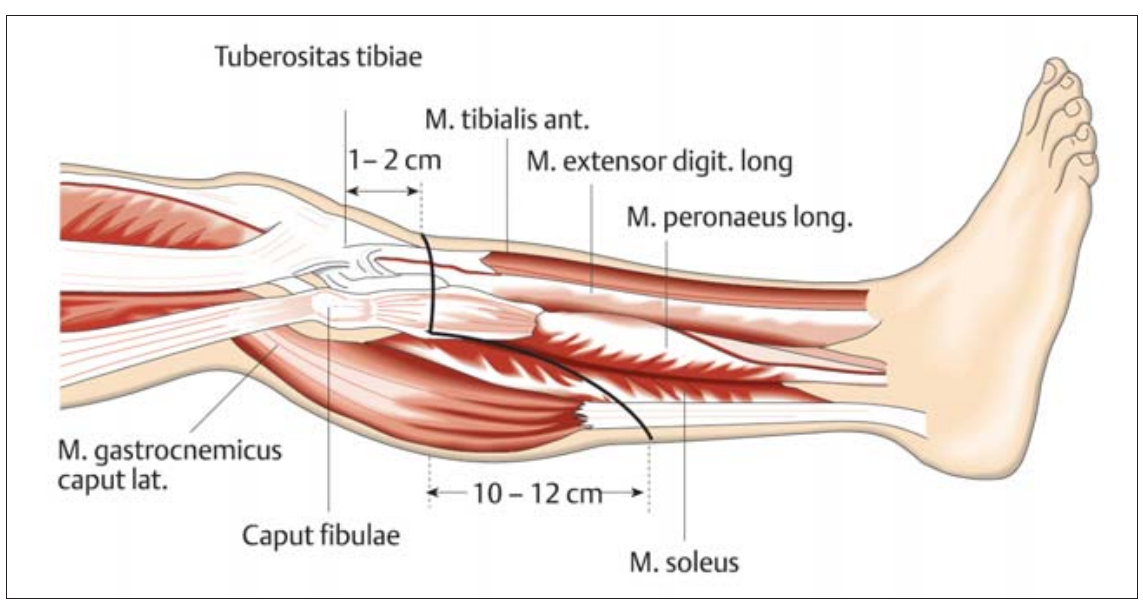

Abb. 17 Schnittführung nach Brückner, 1984, 1992 - mit nur geringen Abweichungen von der Schnittführung nach Burgess. - Aus: Brückner L: Die standardisierte Unterschenkelamputation nach Brückner bei chronisch arterieller Verschlusskrankheit im Stadium IV nach Fontaine, Operat Orthop Traumatol 1992; 4: $63-72$ [4].

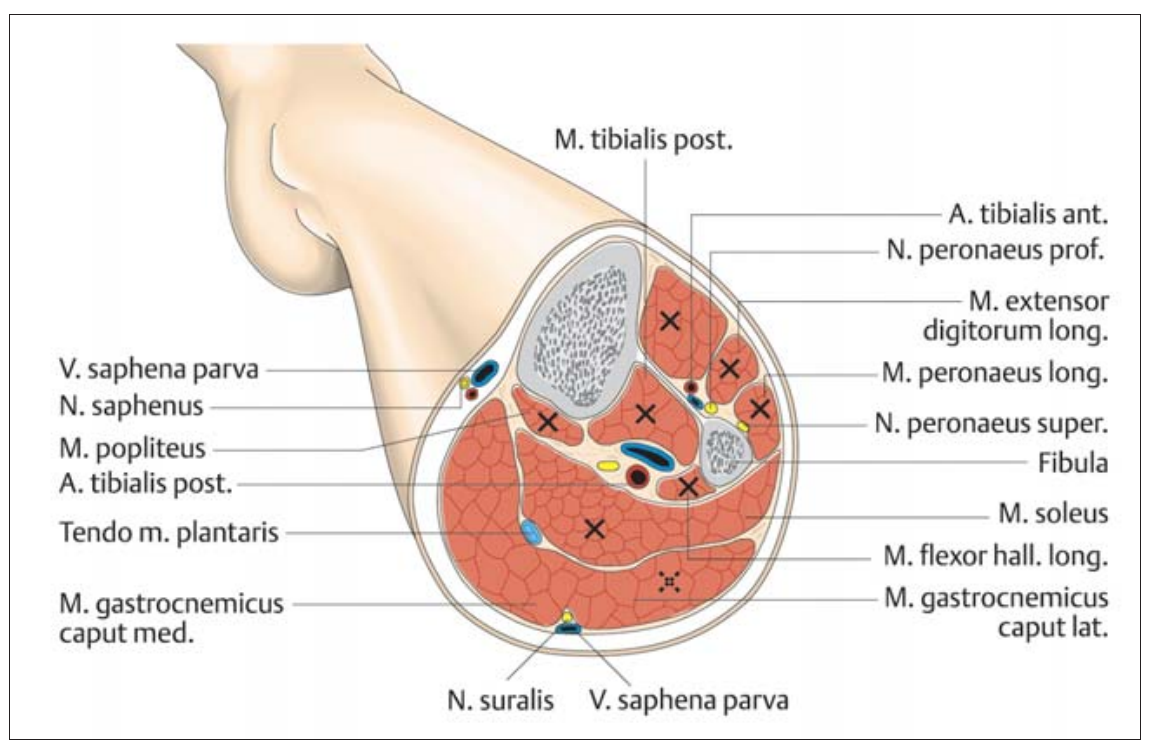

Abb. 18 Standardisierte Amputationstechnik nach Brückner (1992). Die mit Kreuz markierte Muskulatur und die Fibula werden vollständig entfernt; der M. gastrocnemius laterales wird teilweise bis ganz entfernt, je nach ausreichend lateraler Stumpfabdeckungsmöglichkeit. - Aus: Brückner L: Die standardisierte Unterschenkelamputation nach Brückner bei chronisch arterieller Verschlusskrankheit im Stadium IV nach Fontaine, Operat Orthop Traumatol 1992; 4: 63 - 72 [4].

genügend oder gar keine Muskeln entfernt werden. Damit wird gegen das chirurgische Prinzip der Entfernung von nekroseverdächtigem Gewebe verstoßen.

Wir haben anhand klinischer Prüfung, Laboruntersuchungen, Funktionstests, histo- und biochemischer Muskeluntersuchung und ultrastruktureller Beurteilung der Kapillaren die großen Schwierigkeiten in der subjektiven Einschätzung der Unterschenkelmuskulatur nachweisen können. Aufgrund unserer Erfahrungen stellten wir eine „biologi- sche Wertigkeit“" der Muskulatur fest. Dabei kann der M. gastrocnemius medialis unter den Bedingungen der chronisch arteriellen Verschlusskrankheit im Stadium IV nach Fontaine als der Muskel mit der am längsten bestehenden Lebensfähigkeit und der M. tibialis anterior mit der größten Gefährdung seiner Lebensfähigkeit angesehen werden.

Deshalb sahen Robinson (1995) [13] und wir bei dieser Operationsmethode Wundheilungsstörungen besonders im Bereich des M. tibialis anterior.

\section{Kurzer Unterschenkelstumpf (proximales Drittel)}

Standardisierte Unterschenkelamputation nach Brückner $(1984,1992)$

\section{Prinzip}

Plastisches Amputationsverfahren zur Entfernung eines nicht mehr ausreichend durchbluteten und stark schmerzenden peripheren Abschnittes des Unterschenkels bzw. des Fußes.

Die Amputation stellt eine Weiterentwicklung der Unterschenkelamputation nach Burgess dar. Sie sieht die vollständige Entfernung der Fibula und aller Muskeln bis auf den M. gastrocnemius medialis sowie Teile des M. gastrocnemius lateralis vor.

\section{Indikation}

- Chronisch arterielle Verschlusskrankheit im Stadium IV nach Fontaine, wenn - ein gefäßchirurgischer Eingriff keinen Erfolg mehr verspricht, oder - ein gefäßchirurgischer Eingriff ohne Erfolg blieb.

- Trauma

- Infektion

- Tumor

\section{Kontraindikation}

- Entzündung und/oder Gangrän im direkten Amputationsgebiet

- Fixierte Kniebeugekontraktur von $25^{\circ}$ und mehr (Alternative: Knieexartikulation).

\section{Vorteile}

- Erhaltung des Kniegelenkes

- Wegfall der intraoperativen subjektiven Beurteilung der Unterschenkelmuskulatur nach Burgess

- Technisch relativ einfacher Eingriff

- Einhaltung des chirurgischen Prinzips zur Verhütung eines Gasbrandes vollständige Entfernung des minder durchbluteten Gewebes

- Durch primären Verschluss der Wunde keine flächenhaften Narben

- Die Belastung des Patienten durch die Operation ist relativ gering.

- Der Eingriff weist eine ungewöhnlich hohe Knieerhaltungssquote auf.

- Die Operation ist standardisiert und demzufolge überall durchführbar.

- Die prothetische Versorgung ist mit Vollkontaktschaft und Kurzprothese gut möglich. 


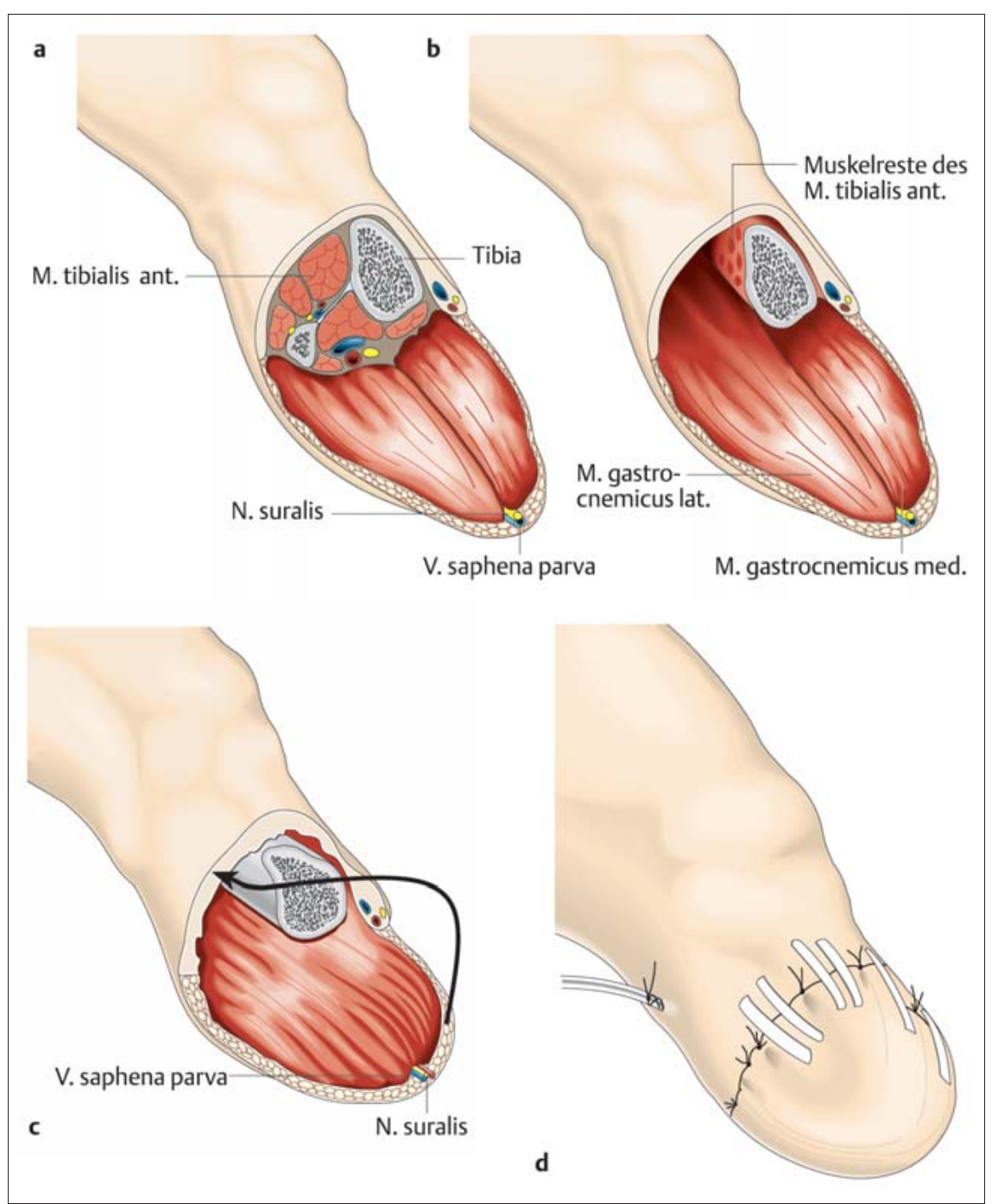

Abb. 19a bis d a Die fibulare Muskelgruppe wird vollständig entfernt. Dadurch entsteht eine Höhle lateral der Tibia, die entweder nur durch den M. gastrocnemius mediales bzw. zusätzlich durch Teile des M. gastrocnemius laterales verschlossen werden muss (b). c Der M. gastrocnemius mediales wird ventral über die abgerundete Tibiaknochenkante nach lateral zur Schließung der entstandenen Höhle geschlagen. Die Muskulatur wird am Periost der lateralen Tibiakante von dorsal nach ventral unter Schonung der Periostgefäße angenäht. d Hautverschluss mit sehr wenigen Nähten; dafür die Verwendung von Steri strips. - Aus: Brückner L: Die standardisierte Unterschenkelamputation nach Brückner bei chronisch arterieller Verschlusskrankheit im Stadium IV nach Fontaine, Operat Orthop Traumatol 1992; 4: 63 - 72 [4].

Nachteile

- Die Amputationshöhe ist am Übergang vom mittellangen zum Kurzstumpf, eher Kurzstumpf.

- Je kürzer der Stumpf, desto weniger Hebelarm ist vorhanden.

\section{Anästhesie}

Wir bevorzugen eine rückenmarksnahe Anästhesie.

Der Patient atmet postoperativ besser und schläft auf der Basis der Zerebralsklerose nicht so lange nach. Darüber hinaus ist diese Anästhesieform eine gute Phantomschmerzprophylaxe.

Cave: Antikoagulanzientherapie

Technik

Ventral liegt der Hautschnitt (1)-2(3) $\mathrm{cm}$ distal der Tuberositas tibiae.

Es wird zirkulär bis auf Höhe der Fibula inzidiert. Am Unterrand der Fibula wird mit dem Amputationsmesser im Durchstichverfahren eingegangen und der dorsale Haut-Muskel-Lappen gebildet. Danach osteotomieren wir die Tibia und die Fibula mit der Amputationssäge nach Bier auf gleicher Ebene (Abb. 17).

Mit der Säge wird die ventrale Tibiakante gut angeschrägt und mit der Knochenfeile geglättet.

Darstellung der in der Regel nicht oder nur minimal blutenden großen Gefäße; deren Unterbindung mit resorbierbarem Nahtmaterial. Der N. tibialis und der N. peronaeus werden bis proximal des Tibiaplateaus verfolgt und scharf durchtrennt. Der N. suralis und der N. saphenus werden gleichfalls weit proximal scharf reseziert.

Die in Abb.18 mit Kreuz unterlegten Muskeln und die Fibula werden total entfernt.

Der M. gastrocnemius lateralis (unterbrochenes Kreuz) wird in Abhängigkeit von der Stumpfdeckung teilweise bis vollständig entfernt.

In Abb.19a stellt sich der Operationssitus nach Absetzung des Unterschenkels dar. Anschließend wird der M. soleus wegen der Gefahr der Thrombenbildung in seinen Venen entfernt. Abb. 19 b zeigt den Operationssitus nach Entfernen der Fibula und der gesamten Muskulatur, außer den beiden Gastrocnemii. Die ventrale Tibiakante ist angeschrägt.

Die am lateralen Periost der Tibia noch vorhandenen Muskelfasern der fibularen Muskelgruppe müssen vorsichtig ohne Verletzung des Periosts mit dem scharfen Löffel entfernt werden.

Der M. gastrocnemius medialis wird über die Tibiastumpfspitze nach lateral unter physiologischer Vorspannung umgeschlagen und möglichst bis zum ehemaligen Ursprung des $M$. tibialis anterior geführt (Abb.19c). Es wird ein Redon-Drain eingelegt und lateral in Höhe des ehemaligen Fibulaköpfchens herausgeführt. Der Muskel wird zuerst ventral am Periost der Tibia durch 2 resorbierbare Fäden fixiert. Eine Hand des Assistenten unterstützt den Haut-Muskel-Lappen, um eine Zugwirkung nach dorsal zu verhindern.

Der Muskel wird dann von dorsal nach ventral mit $2 \times 0$ - bis $3 \times 0$-Fäden vorsichtig am lateralen Periost der Tibia unter Schonung der Periostgefäße fixiert.

Durch dieses enge Vernähen am Knochen wird eine Höhlenbildung verhin- 


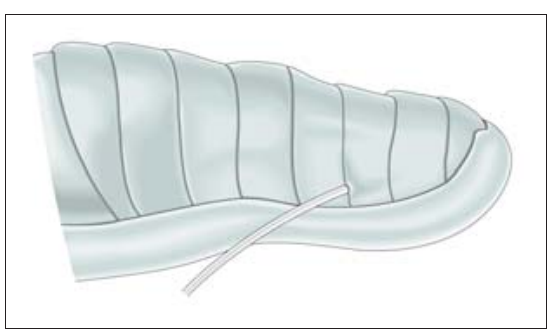

Abb. 20 Postoperative Nachbehandlung mit Wattepolsterung des Stumpfes und bei chronisch arterieller Verschlusskrankheit elastische Wickelung ohne Druck; bei Kontrakturgefahr dorsale Gipsliegeschale. - Aus: Brückner L: Die standardisierte Unterschenkelamputation nach Brückner bei chronisch arterieller Verschlusskrankheit im Stadium IV nach Fontaine, Operat Orthop Traumatol 1992; 4: $63-72[4]$.

dert. Sollte der M. gastrocnemius medialis nicht für die vollständige laterale Stumpfdeckung ausreichen, muss eine vorhandene Lücke noch mit einem Teil des $M$. gastrocnemius lateralis gedeckt werden.

Die Haut wird atraumatisch mit so wenig wie möglich adaptierenden, nicht resorbierbaren Nähten verschlossen. Der übrige Wundrand wird zur Verhinderung von Wundrandnekrosen mit Steri strips adaptiert. Dadurch wird die Durchblutung der Haut am geringsten gestört (Abb.19d).

Cave: Die Muskulatur muss unter physiologische Vorspannung über die ventrale Tibiakante gelegt werden.

Man muss sich also ein Gefühl für die physiologische Vorspannung der Muskulatur erarbeiten.

Nur durch die physiologische Vorspannung der Muskulatur wird im weiteren Verlauf die muskuläre Atrophie weitestgehend verhindert und die Durchblutung des Muskels verbessert. Des Weiteren wird so die Propriozeption am wenigsten gestört und das hat guten Einfluss auf die Verhinderung von Phantomschmerzen $[5,15]$.

\section{Postoperative Behandlung (Abb. 20)}

- Sterile Abdeckung

- Verband mit sterilen Zellstoffbinden: Schutz vor Druck

- Elastische Wickelung: Die elastische Binde wird bei chronisch arterieller Verschlusskrankheit nur um den Stumpf gelegt und nicht straff gezogen.
- Bei Gefahr einer Kniebeugekontraktur wird eine dorsale Gipslongette für die Dauer bis zu 3 Wochen angelegt.

\section{Ergebnisse}

Wir konnten mit dieser standardisierten Operationstechnik in rd. 93\% der Fälle das Kniegelenk erhalten.

Untersuchungen von Heyde et al. 2001 [10] und Stahel et al. 2006 [14] ergeben eine Favorisierung der Standardisierten Unterschenkelamputation nach Brückner gegenüber der Original-BurgessTechnik.

\section{Ultrakurzer Unterschenkel- stumpf}

\section{Prinzip}

Ein ultrakurzer Unterschenkelstumpf zur Verhinderung einer Knieexartikulation kann gebildet werden, wenn die Tuberositas tibiae mit dem Ansatz des Patellabandes zur Streckfähigkeit des Unterschenkelstumpfes erhalten bleibt.

Doppelseitig ultrakurze Unterschenkelstümpfe erweisen sich wegen der hohen Belastung als schwierig und im gegebenen Fall ist demzufolge auch an eine zumindest einseitige Knieexartikulation mit voller Endbelastbarkeit zu denken.

\section{Allgemeine Nachbehandlungskriterien}

Die postoperative Behandlung eines Unterschenkelstumpfes ist scharf nach der Indikation zur Amputation zu trennen.

- Chronisch arterielle Verschlusskrankheit:

- Postoperative Lagerung: Waagerecht im Bett und im Rollstuhl

- Wickelung: Nur mit geringem bis gar keinem Zug von distal nach proximal ohne Faltenbildung. Anhebung der dorsalen Weichteile.

Cave: Innerhalb von 12 Std. kann es bei Durchblutungsstörungen und inadäquatem Druck auf die Haut zu stumpfgefährdenden Nekrosen kommen.

- Amputationen aus anderer Ursache

- Postoperative Lagerung: Stumpf erhöht lagern, um dem Ödem Abfluss zu verschaffen.

- Wickelung: Von distal nach proximal mit abnehmendem Druck. Die elastische Binde wird nach jeder Stumpfumdrehung etwas angezogen. So kann der Druck dosiert wer- den. Die Wickelung erfolgt auch hier von hinten nach vorn bzw. schräg über die Seiten des Stumpfendes, wenn seitliche „Ohren“ am Stumpf vorhanden sind. Der Sinn des Wickelns ist die Ödemprophylaxe und die Stumpfformung. Cave: Schmerzangaben ernst nehmen und evtl. neu wickeln.

\section{Literatur}

1 Baumgartner R, Botta P. Amputation und Prothesenversorgung der unteren Extremität 2. neu bearbeitete und erweiterte Aufl. Stuttgart: Enke, 1995

2 Bohne WHO. Atlas of Amputation Surgery. Stuttgart, New York: Thieme, 1987

3 Brückner L. Die Bestimmung der Amputationshöhe und die Technik der Unterschenkelamputation bei der chronisch arteriellen Verschlusskrankheit im Stadium IV nach Fontane. Habil. Universität Leipzig, 1984

${ }^{4}$ Brückner L. Die standardisierte Unterschenkelamputation nach Brückner bei chronisch arterieller Verschlusskrankheit im Stadium IV nach Fontaine. Operat Orthop Traumatol 1992; 4: 186-195

5 Brückner L, Adler T. Der Muskel: Eine Ursache für Stumpf- und Phantomschmerzen. Orthopädie-Technik 2007; 58: 410-418

6 Burgess EM. The below-knee amputation. Bull Prosthet Res 1968; 10-9: 19-25

7 Dederich R. Amputation der Gliedmaßen. Operationstechnik und prothetische Versorgung. Stuttgart, New York: Thieme, 1987

8 Gottschalk F. Die Biomechanik von Oberschenkel-Amputationen. Orthopädie-Technik 2006; 57: 164 - 169

9 Heller G, Günster C, Swart E. Über die Häufigkeit von Amputationen unterer Extremitäten in Deutschland. Dtsch Med Wochenschr 2005; 130: 1689-1690

${ }^{10}$ Heyde C, Jungmichel D, Neumann W. Majoramputationen bei arterieller Durchblutungsstörung an der unteren Extremität: klinische und frühfunktionelle Ergebnisse. Orthopädie-Technik 2001; 52: 552-557

11 Koller A, Hafkemeyer U, Wetz HH. SymeAmputation bei Fersendefekten. Der Orthopäde 2001; 30: 145-149

12 Pinto MAGS, Harris WW, Baumgartner R. Knochenbrücke mit Fibulasegment bei transtibialen Amputationen. Med Orthop Techn (MOT) 2006; 126: 45-49

13 Robinson KP. Schrägsagittale Unterschenkel-Lappen-Amputation. Orthopädie-Technik 1995; 46: 42-49

14 Stahel PF, Oberholzer A, Morgan SI, Heyde C. Concepts of transtibial Amputation: Burgess technique versus modified Brückner procedure. ANZ J Surg 2006; 76: $942-946$

15 Weiss T, Miltner WHR, Brückner L. Der Phantomschmerz in Abhängigkeit von der Funktionalität der Muskulatur des Stumpfes. Orthopädie-Technik 2006; 57: 904-907

\section{Priv.-Doz. Dr. med. habil. Lutz Brückner}

Chefarzt der Orthopädischen Abteilung

Moritz-Klinik GmbH \& Co. KG Hermann-Sachse-Straße 46 07639 Bad Klosterlausnitz 